\title{
THE CONVERGENCE OF THE SERIES OF THE SOLUTION OF THE CAUCHY PROBLEM FOR THE BBGKY HIERARCHY OF EQUATIONS IN MANY-KIND PARTICLE SYSTEMS
}

\author{
Halyna M. Hubal \\ Computer Science and Information Technologies Faculty \\ Lutsk National Technical University \\ 75 Lvivs'ka str., Lutsk 43018, UKRAINE
}

\begin{abstract}
A many-kind particle system is considered in this paper. The convergence of the series of the solution of the Cauchy problem for the BBGKY hierarchy of equations in cumulant representation is proved.
\end{abstract}

AMS Subject Classification: 35Q82

Key Words: BBGKY hierarchy of equations, many-kind particle system, cumulant representation, interaction region, interaction potential

\section{Introduction}

The non-equilibrium state of many-kind particle systems can be described by a solution of the Cauchy problem for the BBGKY hierarchy of equations. A solution of the Cauchy problem for the BBGKY hierarchy of equations can be represented as the iteration series or the functional series $[1,2,3,4]$. We can describe the cluster nature of the evolution of many-kind particle systems by the solution in the cumulant representation $[3,4]$.

Received: May 14, 2016

Published: August 16, 2016 (c) 2016 Academic Publications, Ltd.

url: www.acadpubl.eu 


\section{Formulation of the Problem}

Consider a many-kind particle system. Denote by $2 \sigma_{i}>0$ and $m_{i}>0$ the $i$-th particle diameter and mass respectively. Particles are characterized by their phase coordinates $\left(q_{i}, v_{i}\right) \equiv x_{i}$. Let particles interact via the pair short-range hard core interaction potential $\Phi(q)$. For the configurations of this particle system, we have the inequalities $\left|q_{i}-q_{i+1}\right| \geq \sigma_{i}+\sigma_{i+1}, i \in \mathbb{Z}^{1} \backslash\{0\}$. The set

$$
W_{n}=\left\{\left(q_{-n_{2}}, \ldots, q_{n_{1}}\right) \in \mathbb{R}^{n_{1}+n_{2}}:\left|q_{i+1}-q_{i}\right|<\sigma_{i+1}+\sigma_{i}\right.
$$

at least for one pair

$$
\left.(i, i+1) \in\left(\left(-n_{2},-n_{2}+1\right), \ldots,(-1,1), \ldots,\left(n_{1}-1, n_{1}\right)\right)\right\}
$$

is called the set of forbidden configurations.

The interaction potential $\Phi(q)$ satisfies the stability condition:

$$
\sum_{i=-n_{2}}^{n_{1}-1} \Phi\left(q_{i}-q_{i+1}\right) \geq-B\left(n_{1}+n_{2}\right), \quad B>0 .
$$

The interaction potential $\Phi(q)$ also satisfies the condition:

$$
\left|\sum_{i=-n_{2}}^{n_{1}-1} \Phi\left(q_{i}-q_{i+1}\right)\right| \leq B\left(n_{1}+n_{2}\right) .
$$

Consider the Banach space $E_{\xi, \beta}$ of sequences

$$
f=\left\{f_{n}\left(x_{-n_{2}}, \ldots, x_{n_{1}}\right)\right\}_{n=n_{1}+n_{2} \geq 0}
$$

of bounded measurable functions $f_{n}\left(x_{-n_{2}}, \ldots, x_{n_{1}}\right)$ equal to zero on the set of forbidden configurations with the norm

$$
\|f\|=\sup _{n \geq 0} \xi^{-n} \sup _{\substack{n_{1}, n_{2} \geq 0 \\ n_{1}+n_{2}=n}} \sup _{x_{-n_{2}}, \ldots, x_{n_{1}}}\left|f_{n}\left(x_{-n_{2}}, \ldots, x_{n_{1}}\right)\right| e^{\beta \sum_{i=-n_{2}}^{n_{1}} \frac{m_{i} v_{i}^{2}}{2}},
$$

where $\xi, \beta>0$.

A solution

$$
F(t)=\left\{F_{|Y|}(t, Y)\right\}_{|Y|=s=s_{1}+s_{2} \geq 0}
$$


of the Cauchy problem for the BBGKY hierarchy of equations is represented as the expansion in the Banach space of sequences of integrable functions [4]:

$$
F_{|Y|}(t, Y)=\sum_{n_{1}=0}^{\infty} \sum_{n_{2}=0}^{\infty} \int_{\left(\mathbb{R}^{1} \times \mathbb{R}^{1}\right)^{n_{1}+n_{2}}} d(X \backslash Y) \mathfrak{A}_{\left(n_{2}, n_{1}\right)}\left(t, X_{Y}\right) F_{|X|}(0, X)
$$

where

$$
\begin{aligned}
Y & =\left(x_{-s_{2}}, \ldots, x_{s_{1}}\right), \\
X & =\left(x_{-\left(n_{2}+s_{2}\right)}, \ldots, x_{s_{1}+n_{1}}\right), \\
d(X \backslash Y) & =d x_{-\left(n_{2}+s_{2}\right)} \ldots d x_{-\left(s_{2}+1\right)} d x_{s_{1}+1} \ldots d x_{s_{1}+n_{1}}, \\
X_{Y} & =\left(x_{-\left(n_{2}+s_{2}\right)}, \ldots, x_{-\left(s_{2}+1\right)}, Y, x_{s_{1}+1}, \ldots, x_{s_{1}+n_{1}}\right), \\
\mathfrak{A}_{\left(n_{2}, n_{1}\right)}\left(t, X_{Y}\right) & =\sum_{P: X_{Y}=\cup_{i} X_{i}}(-1)^{|P|-1} \prod_{X_{i} \subset P} S_{\left|X_{i}\right|}\left(-t, X_{i}\right),
\end{aligned}
$$

$\sum_{P: X_{Y}=\cup_{i} X_{i}}$ is the sum over all ordered partitions of the partially ordered set $X_{Y}$ into $|P|$ nonempty pairwise disjoint partially ordered subsets and the set $Y$ lies in one of the subsets $X_{i}$.

\section{The Convergence of the Series of the Solution of the Cauchy Problem for the BBGKY Hierarchy of Equations}

We prove that the series (2) converges uniformly on $Y$ on every compact set for a finite integration region in the space $E_{\xi, \beta}$.

The following theorem holds.

Theorem 1. For the pair short-range hard core interaction potential $\Phi(q)$ and for the initial data $F(0) \in E_{\xi, \beta}$, the series (2) converges uniformly on $Y$ on every compact set in the space $E_{\xi, \beta}$ if

$$
0 \leq t<t_{0} \equiv \frac{1}{2} \sqrt{\frac{1}{d}\left(\frac{1}{4 \xi} \sqrt{\frac{\beta}{2 \pi}} e^{-\left(2 \beta B+\frac{1}{\sqrt{m}}\right)}-R\right)}
$$

Proof. The integrand of the solution (2) has the form for $n_{1}=n_{2}=0$ :

$$
\mathfrak{A}_{(0,0)}(t, Y) F_{|Y|}(0, Y)=S_{|Y|}(-t, Y) F_{|Y|}(0, Y) .
$$


Let us estimate the integrand (3) by using the stability condition and the condition (1) for the interaction potential:

$$
\begin{gathered}
\left|S_{|Y|}(-t, Y) F_{|Y|}(0, Y)\right| \leq\left|S_{|Y|}(-t, Y)\right|\|F(0)\| e^{-\beta \sum_{i=-s_{2}}^{s_{1}} \frac{m_{i} v_{i}^{2}}{2}} \xi^{s} \leq \\
\leq\|F(0)\| e^{\beta B s} e^{\beta \sum_{i=-s_{2}}^{s_{1}-1} \Phi\left(q_{i}-q_{i+1}\right)} e^{-\beta \sum_{i=-s_{2}}^{s_{1}} \frac{m_{i} v_{i}^{2}}{2}} \xi^{s} \leq \\
\leq\|F(0)\| e^{2 \beta B s} e^{-\beta \sum_{i=-s_{2}}^{s_{1}} \frac{m_{i} v_{i}^{2}}{2}} \xi^{s} .
\end{gathered}
$$

We denote an interaction region by $\Omega_{n_{1}+n_{2}}$, an uninteraction region by $G$ in configuration variables.

The integral (2) is equal to zero in the region $G$.

Indeed, not a single point of $x_{-\left(n_{2}+s_{2}\right)}, \ldots, x_{-\left(s_{2}+1\right)}, x_{s_{1}+1}, \ldots, x_{s_{1}+n_{1}}$ not interacting with each other and with the cluster $Y$ in the region $G$, we obtain such an equality:

$$
\begin{aligned}
\sum_{P: X_{Y}=\cup_{i} X_{i}} & (-1)^{|P|-1} \prod_{X_{i} \subset P} S_{\left|X_{i}\right|}\left(-t, X_{i}\right)= \\
= & \sum_{P: X_{Y}=\cup_{i} X_{i}}(-1)^{|P|-1} S_{1}\left(-t, x_{-\left(n_{2}+s_{2}\right)}\right) \ldots S_{1}\left(-t, x_{-\left(s_{2}+1\right)}\right) \times \\
& \quad \times S_{s}(-t, Y) S_{1}\left(-t, x_{s_{1}+1}\right) \ldots S_{1}\left(-t, x_{s_{1}+n_{1}}\right)=0 .
\end{aligned}
$$

Thus, the integral (2) is equal to zero beyond the interaction region $\Omega_{n_{1}+n_{2}}$. Let us estimate the integral (2), using the inequality (4):

$$
\begin{aligned}
& \left|\int_{\Omega_{n_{1}+n_{2}} \times \mathbb{R}^{n_{1}+n_{2}}} d(X \backslash Y) \mathfrak{A}_{\left(n_{2}, n_{1}\right)}\left(t, X_{Y}\right) F_{|X|}(0, X)\right| \leq \\
& \leq \int_{\Omega_{n_{1}+n_{2}} \times \mathbb{R}^{n_{1}+n_{2}}} d(X \backslash Y) \mid \sum_{P: X_{Y}=\cup_{i} X_{i}}(-1)^{|P|-1} \times \\
& \times \prod_{X_{i} \subset P} S_{\left|X_{i}\right|}\left(-t, X_{i}\right) F_{|X|}(0, X) \mid \leq 2^{n_{1}+n_{2}}\|F(0)\| \times \\
& \times \int_{\mathbb{R}^{n_{1}+n_{2}}} d v_{-\left(n_{2}+s_{2}\right)} \ldots d v_{-\left(s_{2}+1\right)} d v_{s_{1}+1} \ldots d v_{s_{1}+n_{1}} V_{n_{1}+n_{2}}(t) \times
\end{aligned}
$$




$$
\times e^{2 \beta B(n+s)} e^{-\beta \sum_{i=-\left(n_{2}+s_{2}\right)}^{s_{1}+n_{1}} \frac{m_{i} v_{i}^{2}}{2}} \xi^{n+s},
$$

where $V_{n_{1}+n_{2}}(t)$ is the interaction region volume. The estimate of the interaction region volume has the following form [2]:

$$
\begin{aligned}
V_{n_{1}+n_{2}}(t) & \leq \\
\leq & \frac{1}{n_{1} !}\left(\left|l_{s_{1}+s_{2}}(2 t)\right|+2 n_{1}\left(R+4 t^{2} D\right)+4 t \sum_{i=1}^{n_{1}}\left|m_{s_{1}+i} v_{s_{1}+i}\right|\right)^{n_{1}} \times \\
& \times \frac{1}{n_{2} !}\left(\left|l_{s_{1}+s_{2}}(2 t)\right|+2 n_{2}\left(R+4 t^{2} D\right)+4 t \sum_{i=1}^{n_{2}}\left|m_{-\left(s_{2}+i\right)} v_{-\left(s_{2}+i\right)}\right|\right)^{n_{2}},
\end{aligned}
$$

where $\left|l_{s_{1}+s_{2}}(2 t)\right|$ is the length of the interval $l_{s_{1}+s_{2}}(2 t)$ containing $\left(s_{1}+s_{2}\right)$ particles at time $2 t, R$ is the range of the interaction, $D \equiv \sup _{i}\left|F_{i}\right|<+\infty, F_{i}$ is the force acting upon the $i$-th particle by its nearest neighbours.

Therefore,

$$
\begin{aligned}
& \left|\int_{\Omega_{n_{1}+n_{2}} \times \mathbb{R}^{n_{1}+n_{2}}} d(X \backslash Y) \mathfrak{A}_{\left(n_{2}, n_{1}\right)}\left(t, X_{Y}\right) F_{|X|}(0, X)\right| \leq \\
& \leq 2^{n_{1}+n_{2}}\|F(0)\| e^{2 \beta B(n+s)} e^{-\beta \sum_{i=-s_{2}}^{s_{1}} \frac{m_{i} v_{i}^{2}}{2}} \xi^{n+s} \times \\
& \times \int_{\mathbb{R}^{n_{2}}} d v_{-\left(n_{2}+s_{2}\right)} \ldots d v_{-\left(s_{2}+1\right)} e^{-\beta \sum_{i=-\left(n_{2}+s_{2}\right)}^{-\left(s_{2}+1\right)} \frac{m_{i} v_{i}^{2}}{2}} \times \\
& \times \frac{1}{n_{2} !}\left(\left|l_{s_{1}+s_{2}}(2 t)\right|+2 n_{2}\left(R+4 t^{2} D\right)+4 t \sum_{i=1}^{n_{2}}\left|m_{-\left(s_{2}+i\right)} v_{-\left(s_{2}+i\right)}\right|\right)^{n_{2}} \times \\
& \times \int_{\mathbb{R}^{n_{1}}} d v_{s_{1}+1} \ldots d v_{s_{1}+n_{1}} e^{-\beta \sum_{i=s_{1}+1}^{s_{1}+n_{1}} \frac{m_{i} v_{i}^{2}}{2}} \times \\
& \times \frac{1}{n_{1} !}\left(\left|l_{s_{1}+s_{2}}(2 t)\right|+2 n_{1}\left(R+4 t^{2} D\right)+4 t \sum_{i=1}^{n_{1}}\left|m_{s_{1}+i} v_{s_{1}+i}\right|\right)^{n_{1}} .
\end{aligned}
$$

We denote 


$$
\begin{gathered}
A=\int_{\mathbb{R}^{n_{1}}} d v_{s_{1}+1} \ldots d v_{s_{1}+n_{1}} e^{-\beta \sum_{i=s_{1}+1}^{s_{1}+n_{1}} \frac{m_{i} v_{i}^{2}}{2}} \times \\
\quad \times \frac{1}{n_{1} !}\left(\left|l_{s_{1}+s_{2}}(2 t)\right|+2 n_{1}\left(R+4 t^{2} D\right)+4 t \sum_{i=1}^{n_{1}}\left|m_{s_{1}+i} v_{s_{1}+i}\right|\right)^{n_{1}}, \\
\left.\left.B=\int_{\mathbb{R}^{n_{2}}} d v_{-\left(n_{2}+s_{2}\right)} \ldots d v_{-\left(s_{2}+1\right)} e^{-\beta \sum_{i=-\left(n_{2}+s_{2}\right)}^{\frac{m_{i} v_{i}^{2}}{2}} \times} \times \frac{1}{n^{-\left(s_{2}+1\right)}} \times l_{i=1}^{n_{2} !}\left|l_{s_{1}+s_{2}}(2 t)\right|+2 n_{2}\left(R+4 t^{2} D\right)+4 t \sum_{2}+i\right) v_{-\left(s_{2}+i\right)} \mid\right)^{n_{2}} .
\end{gathered}
$$

Thus,

$$
\begin{gathered}
\left|\int_{\Omega_{n_{1}+n_{2}} \times \mathbb{R}^{n_{1}+n_{2}}} d(X \backslash Y) \mathfrak{A}_{\left(n_{2}, n_{1}\right)}\left(t, X_{Y}\right) F_{|X|}(0, X)\right| \leq \\
\leq 2^{n_{1}+n_{2}}\|F(0)\| e^{2 \beta B(n+s)} e^{-\beta \sum_{i=-s_{2}}^{s_{1}} \frac{m_{i} v_{i}^{2}}{2}} \xi^{n+s} A B .
\end{gathered}
$$

We transform the expression $A$ in the form:

$$
\begin{aligned}
A=\sum_{k=0}^{n_{1}} \frac{1}{k !} & \left(\left|l_{s_{1}+s_{2}}(2 t)\right|+2 n_{1}\left(R+4 t^{2} D\right)\right)^{k}(4 t)^{n_{1}-k} \times \\
& \times \sum_{\substack{l_{1}, \ldots, l_{n_{1}}=0 \\
l_{1}+\cdots+l_{n_{1}}=n_{1}-k}}^{n_{1}-k} \frac{1}{l_{1} ! \ldots l_{n_{1}} !} \prod_{i=s_{1}+1}^{s_{1}+n_{1}} m_{i}^{l_{i-s_{1}}} \int_{-\infty}^{\infty} v_{i}^{l_{i-s_{1}}} e^{-\beta \frac{m_{i} v_{i}^{2}}{2}} d v_{i} .
\end{aligned}
$$

We rewrite each integral in (5) in the form

$$
\int_{-\infty}^{\infty} v_{i}^{l_{i-s_{1}}} e^{-\beta \frac{m_{i} v_{i}^{2}}{2}} d v_{i}=\frac{1}{2}\left(\frac{2}{\beta m_{i}}\right)^{\frac{1}{2}\left(l_{i-s_{1}}+1\right)} \Gamma\left(\frac{l_{i-s_{1}}+1}{2}\right) .
$$

Therefore, we estimate the expression $A$ 


$$
\begin{aligned}
A \leq \sum_{k=0}^{n_{1}} \frac{1}{k !}\left(\left|l_{s_{1}+s_{2}}(2 t)\right|+2 n_{1}\left(R+4 t^{2} D\right)\right)^{k}(4 t)^{n_{1}-k} & \\
& \times\left(\frac{2}{\beta}\right)^{n_{1}-\frac{1}{2} k} \pi^{\frac{n_{1}}{2}} 2^{n_{1}-k-1} m_{a}^{-\frac{k}{2}}
\end{aligned}
$$

where $m_{a}=\min \left(m_{s_{1}+1}, \ldots, m_{s_{1}+n_{1}}\right)$.

By analogy, we obtain the estimate of the expression $B$ :

$$
\begin{aligned}
B \leq \sum_{k=0}^{n_{2}} \frac{1}{k !}\left(\left|l_{s_{1}+s_{2}}(2 t)\right|+2 n_{2}\left(R+4 t^{2} D\right)\right)^{k}(4 t)^{n_{2}-k} \times & \\
& \times\left(\frac{2}{\beta}\right)^{n_{2}-\frac{1}{2} k} \pi^{\frac{n_{2}}{2}} 2^{n_{2}-k-1} m_{b}^{-\frac{k}{2}}
\end{aligned}
$$

where $m_{b}=\min \left(m_{-\left(n_{2}+s_{2}\right)}, \ldots, m_{-\left(s_{2}+1\right)}\right)$.

We denote $d=\max \left(D, \frac{2}{R \beta}\right)$. Then holds the inequality (for any $t$ ):

$$
\left(R+4 t^{2} d\right)\left(4 t \sqrt{\frac{2}{\beta}}\right)^{-1} \geq 1 .
$$

Indeed, if $d=\frac{2}{R \beta}$, denoting $\frac{R}{4 t \sqrt{\frac{2}{\beta}}}=\gamma>0$, then this inequality takes on the form $(2 \gamma-1)^{2} \geq 0$. Therefore, if $d=D \geq \frac{2}{R \beta}$, this inequality also holds.

From this inequality follows the inequality:

$$
\left(\frac{\left|l_{s_{1}+s_{2}}(2 t)\right|}{2 n_{1}}+R+4 t^{2} d\right)\left(4 t \sqrt{\frac{2}{\beta}}\right)^{-1} \geq 1 .
$$

Taking into account the inequality (6), we obtain

$$
\begin{aligned}
A \leq \sum_{k=0}^{n_{1}} \frac{1}{k !}( & \left.2 n_{1}\right)^{k}\left(\frac{\left|l_{s_{1}+s_{2}}(2 t)\right|}{2 n_{1}}+R+4 t^{2} d\right)^{k} \times \\
& \times\left(\left(\frac{\left|l_{s_{1}+s_{2}}(2 t)\right|}{2 n_{1}}+R+4 t^{2} d\right)\left(4 t \sqrt{\frac{2}{\beta}}\right)^{-1}\right)^{n_{1}-k} \times \\
& \times(4 t)^{n_{1}-k}\left(\frac{2}{\beta}\right)^{n_{1}-\frac{k}{2}} \pi^{\frac{n_{1}}{2}} 2^{n_{1}-k-1} m_{a}^{-\frac{k}{2}}= \\
= & \sum_{k=0}^{n_{1}} \frac{1}{k !}\left(\frac{n_{1}}{\sqrt{m_{a}}}\right)^{k}\left(\frac{\left|l_{s_{1}+s_{2}}(2 t)\right|}{2 n_{1}}+R+4 t^{2} d\right)^{n_{1}}\left(\frac{2}{\beta}\right)^{\frac{n_{1}}{2}} \times
\end{aligned}
$$




$$
\times \pi^{\frac{n_{1}}{2}} 2^{n_{1}-1} \leq e^{\frac{n_{1}}{\sqrt{m_{a}}}}\left(\frac{\left|l_{s_{1}}+s_{2}(2 t)\right|}{2 n_{1}}+R+4 t^{2} d\right)^{n_{1}}\left(\frac{2 \pi}{\beta}\right)^{\frac{n_{1}}{2}} 2^{n_{1}-1}
$$

By analogy, we obtain the estimate of the expression $B$ :

$$
B \leq e^{\frac{n_{2}}{\sqrt{m_{b}}}}\left(\frac{\left|l_{s_{1}+s_{2}}(2 t)\right|}{2 n_{2}}+R+4 t^{2} d\right)^{n_{2}}\left(\frac{2 \pi}{\beta}\right)^{\frac{n_{2}}{2}} 2^{n_{2}-1} .
$$

We denote $m=\min \left(m_{a}, m_{b}\right)$.

The estimate of the series (2) takes the form:

$$
\begin{aligned}
&\left|F_{|Y|}(t, Y)\right| \leq e^{2 \beta B s}\|F(0)\| \xi^{s} e^{-\beta \sum_{i=-s_{2}}^{s_{1}} \frac{m_{i} v_{i}^{2}}{2}} \times \\
& \times \sum_{n_{1}=0}^{\infty}(2 \xi)^{n_{1}} e^{2 \beta B n_{1}} A \sum_{n_{2}=0}^{\infty}(2 \xi)^{n_{2}} e^{2 \beta B n_{2}} B .
\end{aligned}
$$

Since

$$
\lim _{n_{1} \rightarrow \infty} \frac{\left|l_{s_{1}+s_{2}}(2 t)\right|}{2 n_{1}}=0, \quad \lim _{n_{2} \rightarrow \infty} \frac{\left|l_{s_{1}+s_{2}}(2 t)\right|}{2 n_{2}}=0,
$$

then the series (7) converges if

$$
0 \leq t<t_{0} \equiv \frac{1}{2} \sqrt{\frac{1}{d}\left(\frac{1}{4 \xi} \sqrt{\frac{\beta}{2 \pi}} e^{-\left(2 \beta B+\frac{1}{\sqrt{m}}\right)}-R\right)} .
$$

Thus, for the initial data $F(0) \in E_{\xi, \beta}$, the series (2) converges uniformly on $Y$ on every compact set if the condition (8) is fulfilled.

\section{Conclusions}

We proved the convergence of the series of the solution of the Cauchy problem for the BBGKY hierarchy of equations in the cumulant representation in manykind particle systems.

\section{References}

[1] N. N. Bogolyubov, Problems of a dynamical theory in statistical physics, Gosudarstv. Izdat. Tehn.Teor. Lit., Russian Federation (1946) [in Russian]. 
[2] C. Cercignani, V. I. Gerasimenko, D. Ya. Petrina, Many-particle dynamics and kinetic equations, Kluwer Acad. Publ., Netherlands (1997), doi: http://dx.doi.org/10.1007/97894-011-5558-8.

[3] G. N. Gubal', On the existence of weak local in time solutions in the form of a cumulant expansion for a chain of Bogolyubov's equations of a one-dimensional symmetric particle system, Journal of Mathematical Sciences, 199 (2014), 654-666, doi: http://dx.doi.org/10.1007/s10958-014-1892-1.

[4] V. I. Gerasimenko, T. V. Ryabukha, M. O. Stashenko, On the structure of expansions for the BBGKY hierarchy solutions, J. Phys. A: Math. and General, 37, No. 42 (2004), 98619872, doi: http://dx.doi.org/10.1088/0305-4470/37/42/002. 
